\title{
El aprendizaje colaborativo en las Finanzas mediante Wikis. Aplicación práctica a las Políticas de Dividendos.
}

\section{Alfredo Juan Grau Grau}

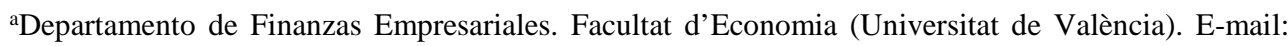
Alfredo.Grau@uv.es.

\begin{abstract}
In this paper we use the Wiki platform to facilitate, among our students, learning a branch of the Corporate Finance: the dividend policy following the listed companies. This is an inexact science, therefore, there are many factors involved in that policy and many skills that students must relate to, so we consider very appropriate the collaborative approach to learning. Developing a Wiki facilitates a more active participation by the student and, consequently, the development of critical thinking that facilitates the understanding of finance. The result of the study shows the main advantage of this method of teaching and learning, student interest and the high degree of participation indirectly translates into better academic results.
\end{abstract}

Keywords: collaborative learning, Wikis, corporate finance, dividend policy.

\section{Resumen}

En este trabajo utilizamos la plataforma Wiki para facilitar, entre nuestros alumnos, el aprendizaje de una rama de las Finanzas Corporativas: la Política de Dividendos que siguen las empresas que cotizan en bolsa. Al no ser una ciencia exacta, son muchos los factores que intervienen en dicha política y muchos los conocimientos que el alumno debe relacionar, por lo que consideramos muy apropiado el enfoque colaborativo para su aprendizaje. La elaboración de una Wiki facilita una participación más activa por parte del estudiante y, por tanto, el desarrollo de un pensamiento crítico que facilita la comprensión de las Finanzas. El resultado del trabajo muestra como principal ventaja de esta metodología de enseñanzaaprendizaje, el interés que suscita en el alumno y su elevado grado de participación que de forma indirecta se traduce en un mayor rendimiento académico..

Palabras clave: aprendizaje colaborativo, Wikis, finanzas corporativas, política de dividendos. 
El aprendizaje colaborativo en las Finanzas mediante Wikis. Aplicación práctica a las Políticas de Dividendos.

\section{Introducción}

En este trabajo presentamos los principales resultados que hemos obtenido al aplicar la plataforma Wiki como método de aprendizaje colaborativo en el proceso enseñanzaaprendizaje de alumnos de educación superior.

Efectivamente se ha comprobado que los estudiantes retienen a las 24 horas un 5\% de los conocimientos adquiridos mediante una lección magistral. Ese porcentaje se incrementa hasta el 50\% si los conocimientos se han adquirido mediante la discusión en grupo y al $75 \%$ con experiencias prácticas (Salinas, 1995) ${ }^{1}$. Por tanto, mediante el aprendizaje colaborativo los alumnos adquirirán más conocimientos además de más competencias.

Respecto a las competencias que debe adquirir el alumno, el EEES (Espacio Europeo de Educación Superior) establece unas directrices para que los estudios superiores consigan desarrollarlas junto con la adquisición de conocimientos. Esas competencias deben ir encaminadas a permitir al estudiante interpretar la información, evaluarla, generar nueva información y tomar decisiones; es decir, desarrollar un pensamiento comprensivo, crítico y creativo. Ante este reto, el docente universitario debe aprovechar las técnicas a su alcance para posibilitar el desarrollo de esas competencias en el alumno. Como señalan Gutiérrez y otros (2013), la Universidad tiene una responsabilidad educativa, que se traduce no sólo en la presentación de los conocimientos sino también en favorecer vías de acceso y la complicidad responsable de los estudiantes en este mundo de conocimientos, dentro de situaciones que favorezcan su participación, crítica y desarrollo de un propio pensar.

En este sentido, el aprendizaje colaborativo puede desarrollarse a través de diversas técnicas. Gracias al desarrollo de las TIC (Tecnologías de la Información y la Comunicación) existe una variada oferta de software libre y gratuito disponible en la red que facilita la aplicación de metodologías docentes enfocadas en el aprendizaje colaborativo como son los blogs, los wikis o las redes sociales.

Estos recursos educativos han crecido exponencialmente (Geser, 2007) y ello se debe al buen funcionamiento de los sistemas basados en el software libre. Como señala Esteve (2009) estas nuevas tecnologías abren la puerta a un nuevo modelo de universidad que favorece la participación, la iniciativa, el espíritu crítico y, en definitiva, el aprendizaje.

Varios autores, como Sáez y Ruiz (2012) y Muñoz y otros (2014), han estudiado el grado de aceptación entre los alumnos universitarios de metodologías de aprendizaje colaborativo o basado en grupos mediante TIC. En dichos estudios se demuestra que estas técnicas son eficaces en el aprendizaje al mostrar una elevada aceptación y una valoración positiva por parte del alumno.

${ }^{1}$ Citado en Muñoz y otros (2014). 
A su vez, resulta imperante formar adecuadamente a profesionales a través de la adaptación de los modelos basados en la enseñanza - aprendizaje (Salinas, 1999).

Desde el EEES, además de las reformas normativas de los países que conforman el proceso de Bolonia, se han realizado actuaciones para su implantación, y para ello, las figuras del profesor y alumno requiere de continuas adaptaciones a sus procesos de enseñanza aprendizaje a través de los nuevos planes de estudio y sobre todo, un adecuada utilización de las metodologías docentes disponibles (Ferro, Martínez y Otero, 2009 y Suriá, 2010).

\section{Objetivos}

Siguiendo el hilo argumental vertido en la introducción, nuestro principal objetivo se traduce en potenciar la participación activa del estudiante, el interés por el aprendizaje de la asignatura y el pensamiento crítico. Todo ello conduce, por una parte, a la adquisición de competencias establecidas por el EEES. Y, por otra parte a una mayor retención de los conocimientos adquiridos.

De entre la oferta de software libre y gratuito que hay disponible en la red, y con el fin de alcanzar los objetivos planteados, hemos elegido las Wikis (particularmente las Wikispaces) como herramienta idónea dado que permite compartir contenidos publicados a la vez que crear, editar y reestructurar esos contenidos de manera flexible (Choy y Ng, 2007 y BenZvi, 2007). El uso y manejo de las Wikispaces no requieren de muchos conocimientos de informática ni de lenguajes de programación. Su funcionamiento es bastante intuitivo y por tanto, la hace especialmente atractiva a la hora de implementarla en el aula (Villalva, 2009).

Las Wikispaces desarrollan una gran diversidad de funciones y es una herramienta especialmente indicada para favorecer el clima de colaboración entre alumno-profesor y alumno-alumno. A su vez permite compartir todo tipo de materiales, documentos, archivos, proyectos, etc. (Bruns y Humphreys, 2005) con un elemento común que es el desarrollo de los contenidos de una misma disciplina.

\section{Desarrollo de la innovación}

\subsection{Filosofía Wiki}

La Wiki se podría definir como una aplicación informática que reside en un servidor web cuya accesibilidad está abierta a cualquier usuario de la red. A través de ella se permite añadir información, opinar sobre sus contenidos, modificar, editar y/o eliminar los mismos. A diferencia de los blogs, por ejemplo, a sus usuarios se les faculta para poder realizar sus contribuciones con el objetivo de que la plataforma mejore en contenidos.

De esta forma, las Wikis proporcionan espacios de trabajo en los que se pone en práctica y se aprovechan las ventajas del enfoque colaborativo. Efectivamente y como señala Guitierrez y Castillo (2012) permiten que los alumnos construyan su propio aprendizaje

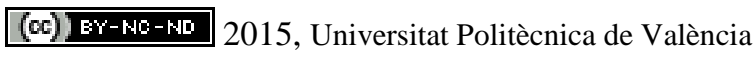


El aprendizaje colaborativo en las Finanzas mediante Wikis. Aplicación práctica a las Políticas de Dividendos.

mediante la interacción con el medio y con los demás alumnos que participan de la Wiki. Se puede llegar a producir un verdadero intercambio de conocimiento, ya que crear Wikis implica editar juntos, adjuntar información para que los compañeros la complementen o la modifiquen en función de unos objetivos comunes de aprendizaje.

A la luz de todo lo dicho, se vislumbra que las Wikis facilitan un espacio web donde varios usuarios puedan crear páginas en la red cuyos contenidos se mueven en torno a una misma temática, de forma que cada uno de ellos puede realizar sus aportaciones a través de sus conocimientos. De esta forma, se va completando de conocimientos la Wiki y consecuentemente una comunidad de usuarios pueden compartir y debatir sobre los contenidos de un mismo tema o categoría. Así, las Wikis pasa a ser una de las primeras herramientas que ofrecen una gran utilidad a la hora de gestionar el conocimiento en red (Rodríguez, 2008).

Seguidamente pasamos a enumerar parte de los motivos que nos han motivado a decantarnos por este recurso como vehículo útil para mejorar el rendimiento académico:

- Aplicación informática de uso libre, de fácil manejo y sencillo. No precisa conocer algoritmos informáticos determinados a la par que, no se exige el conocimiento en el manejo de ningún tipo de lenguaje de programación determinado.

- Su utilización puede ser llevada a cabo de forma simultánea por un gran número de usuarios. Ello permite que queden registrados todos los movimientos efectuados.

- Facilidad y rapidez en el acceso a todas las novedades que han ido incorporando todos los integrantes de la plataforma.

- Se pueden integrar y salvar archivos multimedia (imágenes, videos, elementos, tablas y gráficos, presentaciones, documentos de texto dinámicos, links, etc.

- Se facilita un foro de manera que sus miembros pueden incorporar comentarios y/o leer aquellos que han sido manifestados por el resto de los usuarios.

- Todas las páginas de la Wiki facilitan un historial que registra todas las ediciones. La página es gestionada por un administrado que es quien permite o no, los accesos.

- Dependiente de la configuración preferida por el administrador/creador de la Wikis, pueden ser de uso privado o público.

Respecto a la creación de una Wiki es aconsejable buscar las plataformas disponibles que permitan hacerlo de manera gratuita y libre de espacios publicitarios para poder desarrollar contenidos académicos en el marco de la educación superior. En este trabajo se ha elegido la plataforma Wikispaces porque su servicio web se ajusta perfectamente a nuestras necesidades y objetivos.

(c)) EY-NC-ND 2015, Universitat Politècnica de València 
En la Fig. 1 se aprecia la página de acceso a Wikispaces y las diligencias de registro a la misma (elegir nombre de usuario, proporcionar una dirección de correo electrónico y proporcionar una clave de acceso). Se proporciona dos posibilidades: crear una página para los docentes o para los alumnos.

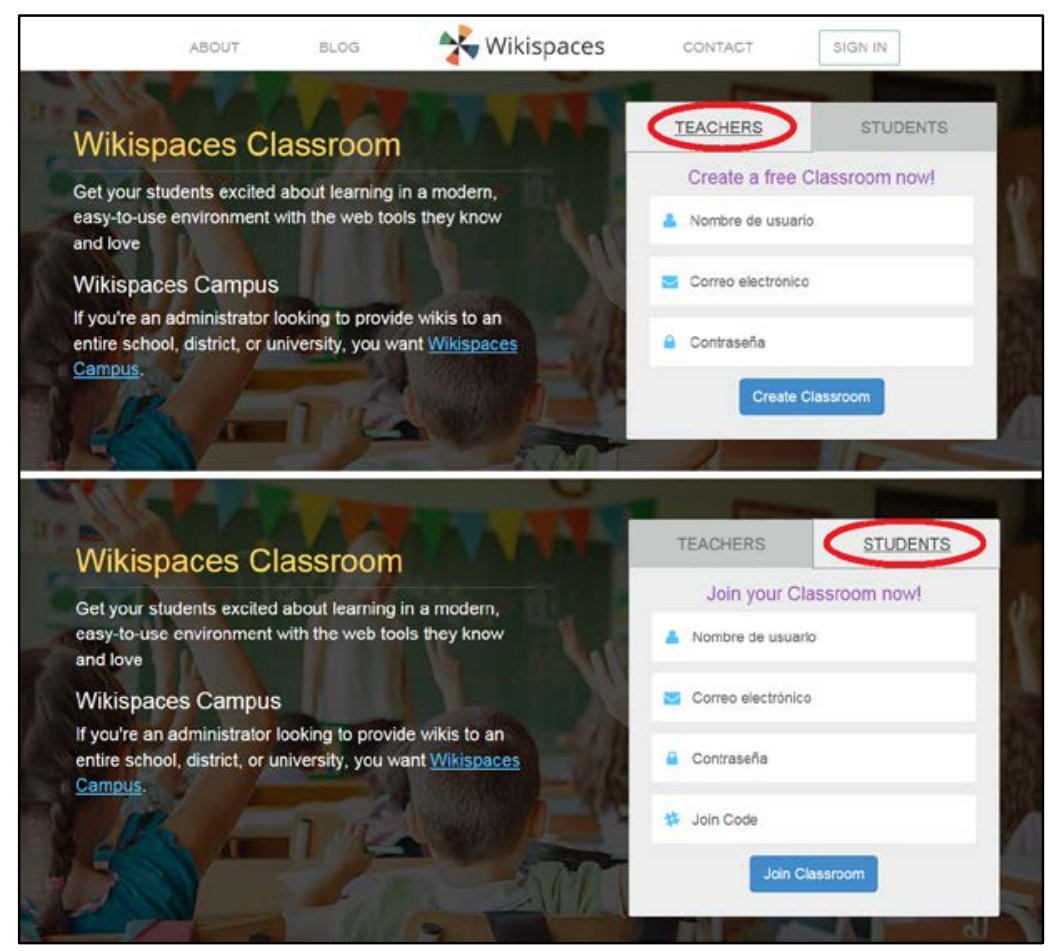

Fig. 1. Página de acceso a Wikispaces

A continuación, la plataforma pide una serie de datos concernientes al usuario como son: el nombre elegido para la Wiki, sus datos personales, nivel educativo, ciudad de residencia, país, etc.).

Referente a la gestión/mantenimiento de la Wiki se recomienda consultar los diferentes tutoriales disponibles en Internet, de entre los cuales destacamos el documento publicado por Barroso (2009).

\subsection{Diseño del experimento docente: Políticas de dividendos en una Wikispace.}

En primer lugar, el profesor (administrador del grupo) facilita todo tipo de información vinculada a los contenidos de Finanzas estudiados en el módulo. Esta información hace referencia a noticias de prensa escrita, noticias en soporte audiovisual, etc.), y se facilita al final de cada tema y estrechamente relacionada con los contenidos que se han desarrollado.

\section{(c)) EY-NC-ND 2015, Universitat Politècnica de València}


El aprendizaje colaborativo en las Finanzas mediante Wikis. Aplicación práctica a las Políticas de Dividendos.

Al realizar la práctica hemos tenido en cuenta cual debe ser el papel del docente que, como señalan Brenes, García y Zarate (2012) se corresponde con el de guía y facilitador del proceso de comunicación y exploración de conocimiento. Su papel es presentar un tema que sirva de introducción. A partir de ahí, debe ser discutido, modificado y editado por la interacción del grupo.

Este experimento lo aplicaremos al aprendizaje de una rama de las Finanzas Corporativas: la Política de Dividendos que siguen las empresas que cotizan en bolsa. Los alumnos a los que va dirigido son los matriculados en el Programa de Máster Oficial de la UV: MBA (Management Business Administration), para el capítulo de Política de Endeudamiento y Dividendos en el curso 2014-2015. Los integrantes de este máster son fundamentalmente ingenieros, y por tanto, en su formación está ausente toda la filosofía que subyace en las Finanzas. Ello nos ha motivado a utilizar esta metodología tan flexible para despertar su interés y sobre todo, para crear un clima que favorezca el debate y la discusión constructiva.

\subsubsection{Planificación y organización}

La primera actividad que requiere la construcción de una Wiki es llevar a cabo una adecuada planificación para que la Wikispaces cumpla con su cometido. Hay que tener en cuenta que el docente. Ello conlleva:

- Determinar cuáles son nuestros objetivos y las competencias profesionales que pretendemos desarrollar con la Wikispaces. En concreto, esas competencias son: despertar el interés sobre las noticias relacionadas con las Finanzas y que se publican tanto en prensa gráfica como en soportes audiovisuales; desarrollar la capacidad crítica a la hora de valorar los contenidos económico-financieros de una noticia; y conocer los cauces y medios alternativos para ampliar la información sobre hechos económicos financieros. Por ejemplo, en el caso de un anuncio sobre la Política de Dividendos de una compañía en una notica de prensa gráfica, tomar la iniciativa de acudir a otras fuentes más directas sobre la misma y que podrían estar disponibles en la CNMV o en la propia web de la sociedad cotizada.

- Localizar gran variedad de materiales gráficos y visuales que serán necesarios e irán componiendo la Wiki. Para el Grupo 3, que es el ejemplo que desarrollamos en el presente trabajo, hemos seleccionado los siguientes materiales:

- Artículos publicados en varios (Cinco Días, El Economista, etc.) que tratan la Política de Dividendos. En este caso “Dividendos elevados y crecientes”.

- Video ilustrativo: “Los problemas del sistema financiero y las políticas de dividendos” por Ángel de Molina (Director de análisis de Tressis S.V.).

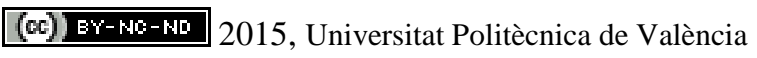

Congreso IN-RED (2015) 
- Clasificación y distribución de toda la información que se va publicar en la Wiki. Para este proyecto se diseñó de forma cronológica todos los eventos que irían incorporando en el espacio web indicando siempre cuál era la tarea solicitada por el profesor.

- Disposición de los elementos integrantes de la Wiki: contenidos, diseño (iconos, imágenes, etc.).

- Funciones de los usuarios. El acceso a la Wiki es de carácter privado y aunque cada cinco miembros conforma un grupo de trabajo, todos pertenecen a un proyecto común y por tanto, todos pueden acceder a los contenidos de todos los grupos.

\subsubsection{Diseño y estructura}

Una vez planificado el proyecto se procede a diseñar y estructura el espacio web. En primer lugar hay que localizar una plataforma en Internet que se ajuste a las exigencias del proyecto. Resulta interesante localizar un soporte informático gratuito y que no incorpore publicidad. En este proyecto se ha seleccionado la herramienta Wikispace.com.

Tal y como se indicó anteriormente, la plataforma Wiki solicita qué tipo de página queremos crear: de tipo académica o resto de posibilidades (Fig. 2).

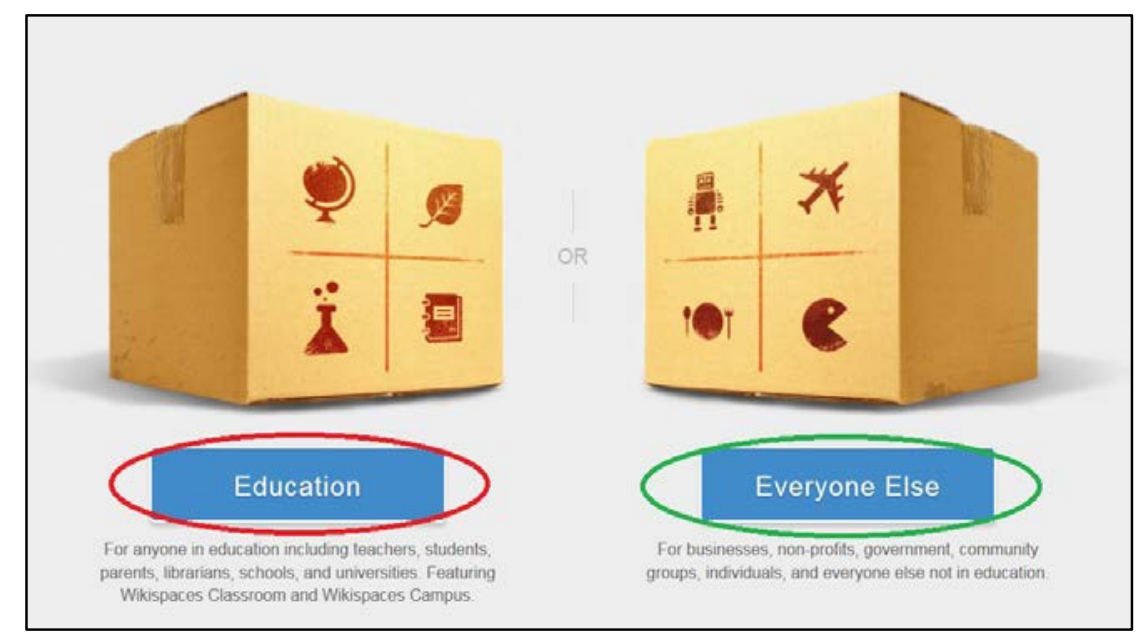

Fig. 2. Web de acceso

Una vez introducidos nuestros datos, nos solicita un nombre para la Wiki. Para nuestra Wiki hemos elegido el nombre "FCMBA".

En Ajustes se nos permite definir/modificar la configuración que por defecto viene asignada: seleccionar nombre, descripción de la Wiki y fundamentalmente la tipología de la aplicación. Hemos seleccionado Classroom que es la que se ajusta mejor al tipo de relación que se quiere establecer entre docente y dicente (Fig. 3).

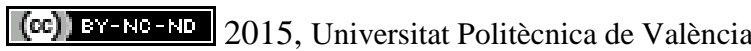


El aprendizaje colaborativo en las Finanzas mediante Wikis. Aplicación práctica a las Políticas de Dividendos.

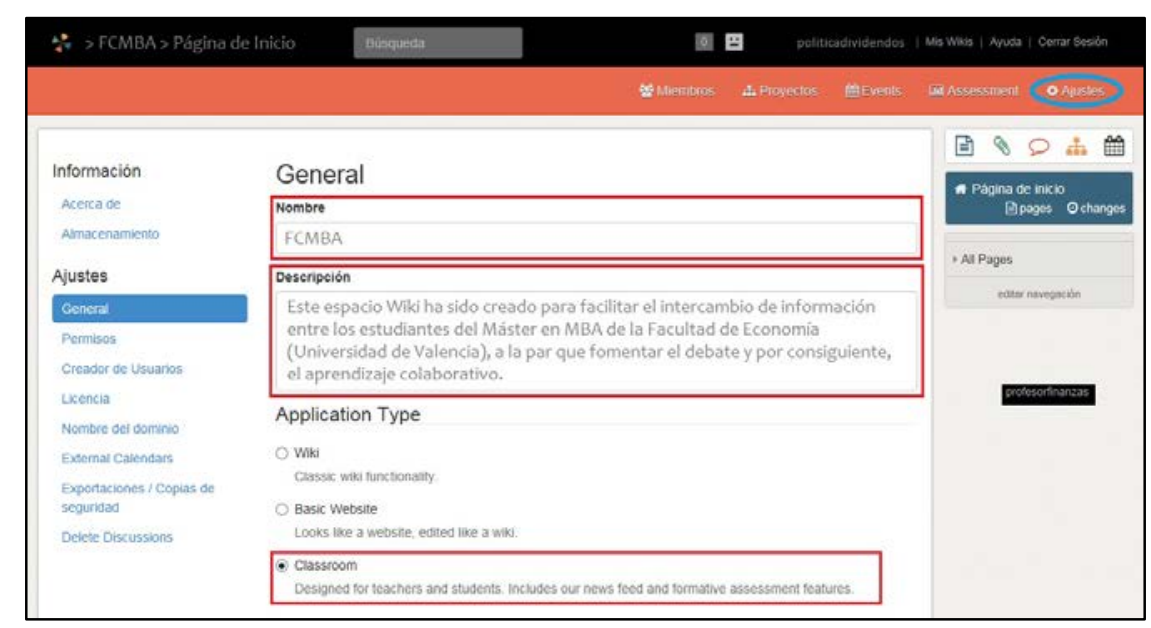

Fig. 3. Configuración general

Después procedemos a configurar los correspondientes Permisos, que son las distintas posibilidades para controlar el acceso de los usuarios. Se ha optado por el acceso "privado" para que así, solo los miembros autorizados por el administrador podrán acceder y participar en la Wiki (Fig. 4).

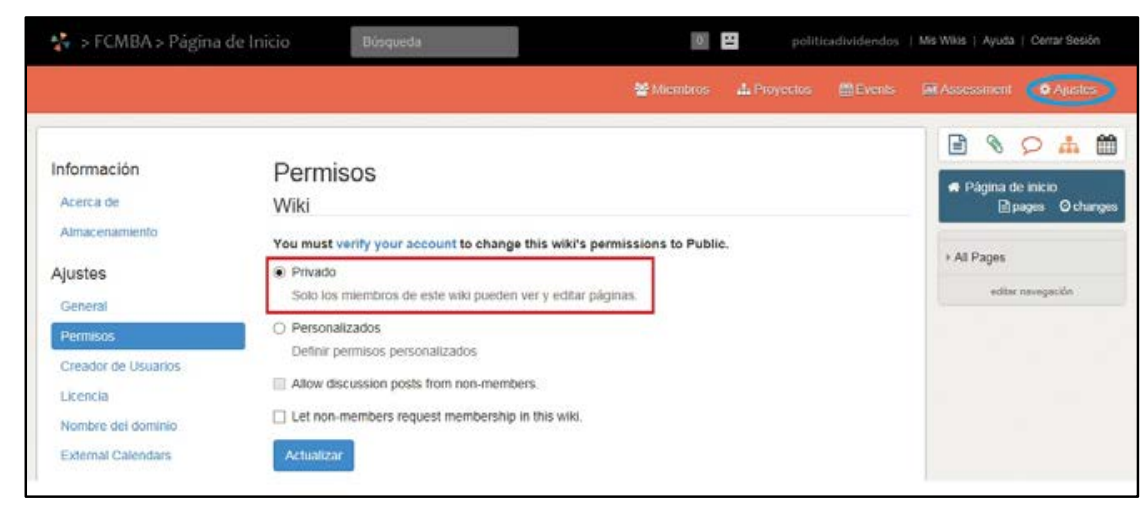

Fig. 4. Permisos de acceso

Los contenidos de la plataforma requieren que el administrador indique qué tipo de tratamiento, sobre el copyright, se quiere seleccionar. Nos hemos decantado por utilizar la licencia de Creative Commons ${ }^{2}$ en su versión Non-Commercial. Esta opción permite a otros usuarios de la Wiki: copiar, distribuir, exhibir, y realizar su trabajo, pero sólo para fines no comerciales (Fig. 5).

\footnotetext{
${ }^{2}$ Para más información onsulte la dirección web:: http://es.creativecommons.org/blog/licencias/.
} 


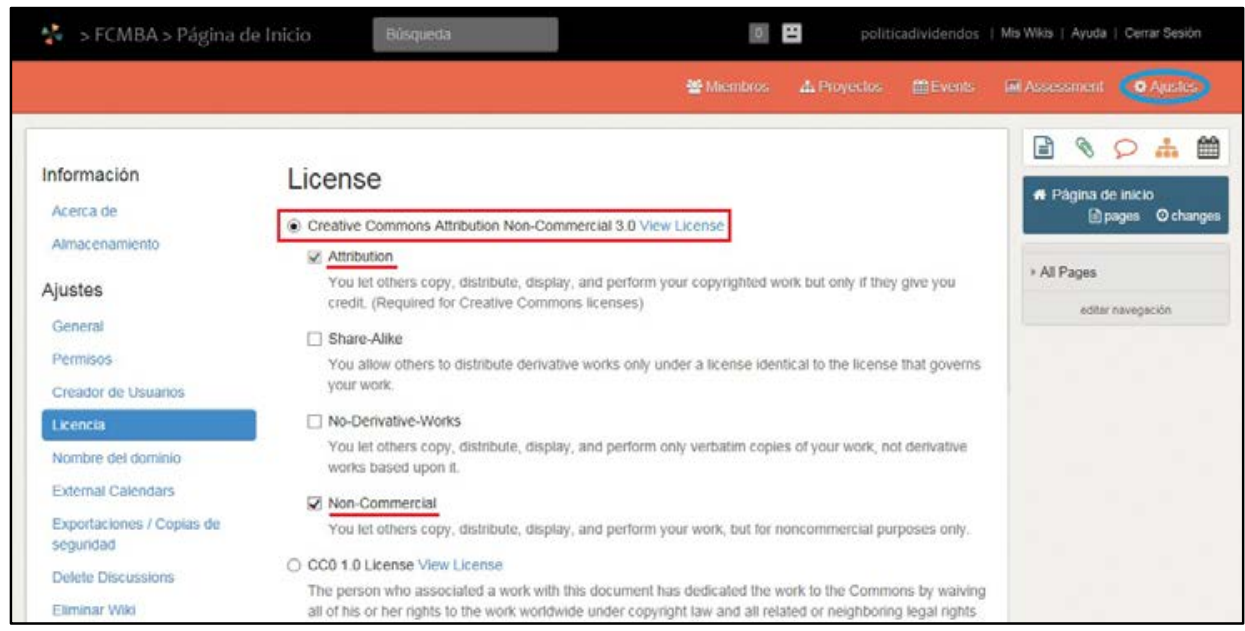

Fig. 5. Licencia seleccionada

\subsubsection{Crear el proyecto y organizar los grupos de trabajo}

En el programa de Máster Oficial MBA, para el módulo de Finanzas el número de alumnos matriculados nos ha permitido abrir cinco grupos cuyos miembros oscilan entre cuatro y cinco alumnos. Cada uno de estos grupos tendrá una tarea diferente asignada al final de cada tema.

Al proyecto se le adjudica el nombre de POLÍTICA DE DIVIDENDOS y en cada uno de los cinco subgrupos, se incorpora material variado sobre las Políticas de Dividendos acometidas por las empresas cotizadas en Bolsa española (Fig. 6).

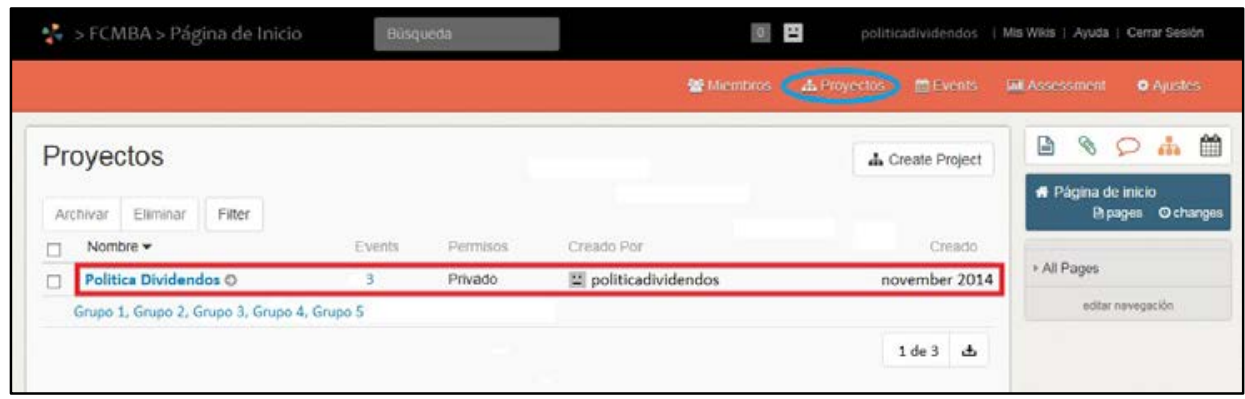

Fig. 6. Configuración del proyecto

A continuación, se invita a los miembros que van a formar cada uno de los distintos grupos (opción 1) o que ellos mismos soliciten la incorporación a la Wiki (opción 2) y desde allí, trasladarlos a un grupo de trabajo determinado (Fig. 7).

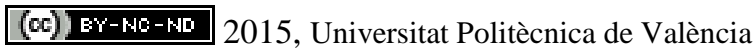

Congreso In-Red (2015) 
El aprendizaje colaborativo en las Finanzas mediante Wikis. Aplicación práctica a las Políticas de Dividendos.

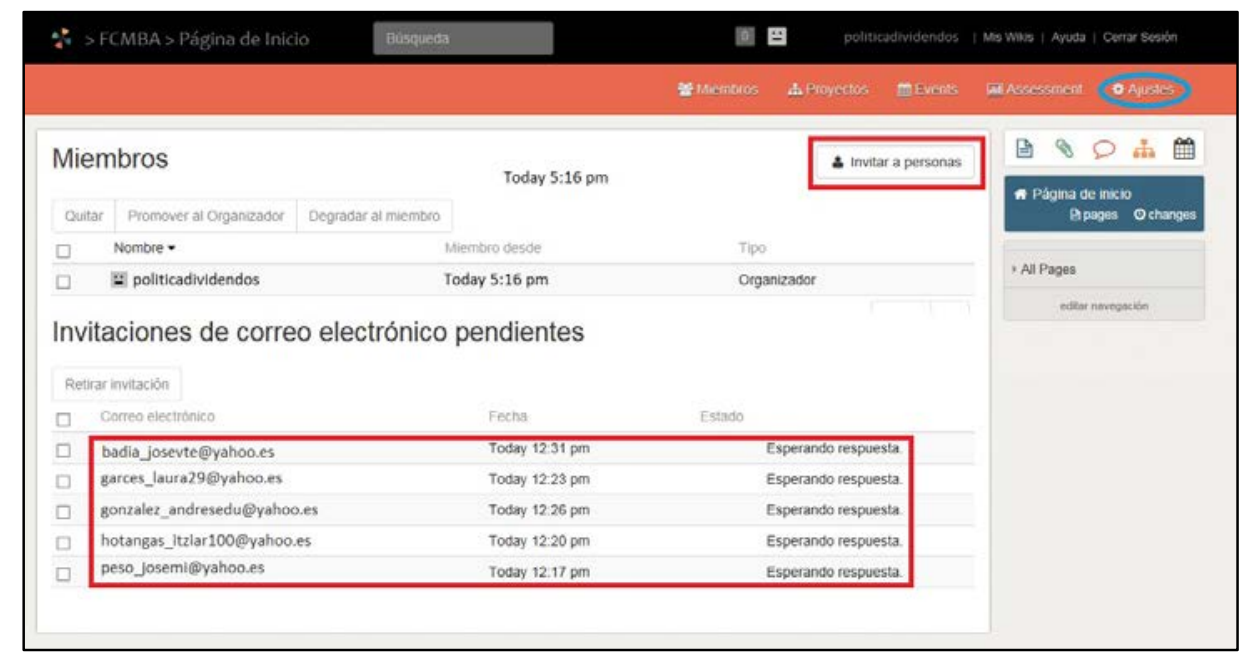

Fig. 7. Gestión de miembros en los grupos

\subsubsection{Proporcionar/integrar los distintos materiales}

El docente va incorporando los materiales docentes (noticias, videos, etc.) en cada uno de los grupos de trabajo creados y que versan sobre las Políticas de Dividendos de las empresas cotizadas, organizadas con el siguiente orden secuencial:

1. Primeramente se diseña un Evento donde se detalla la secuenciación cronológica de los contenidos académicos que se van incorporando sobre la cuestión a debatir, para el caso que nos aborda en el grupo 3, los efectos del reparto de dividendos elevados y crecientes (Véase Anexo I).

2. Se van incorporando más elementos a medida que se van generando. Una vez se publica en la Wiki la primera noticia, se les unos días a los alumnos de cada grupo para que hagan sus comentarios y apreciaciones (Fig. 8) de carácter muy general.

3. Una vez transcurridos los días previstos, el docente plantea cuestiones al hilo de la noticia y espera que los miembros del grupo se posicionen al respecto y pongan en práctica los conocimientos aprendidos.

4. A partir de ese momento, el profesor va facilitando nuevas noticias y materiales diversos que requieren de la participación de los alumnos.

\section{Resultados}

Tras el análisis de los resultados obtenidos, se aprecia claramente un efecto sinérgico en sentido positivo ya que, los alumnos no se han limitado a estudiar y debatir sobre el material proporcionado por el administrador/profesor, sino que, además, han incorporado 
todo tipo de materiales que han ayudado a reforzar y ampliar los contenidos iniciales. Por ejemplo, otro tipo de noticias publicadas para otras empresas, videos descargados de youtube.com donde se hacen análisis sobre los efectos de las distintas políticas de dividendos, etc.

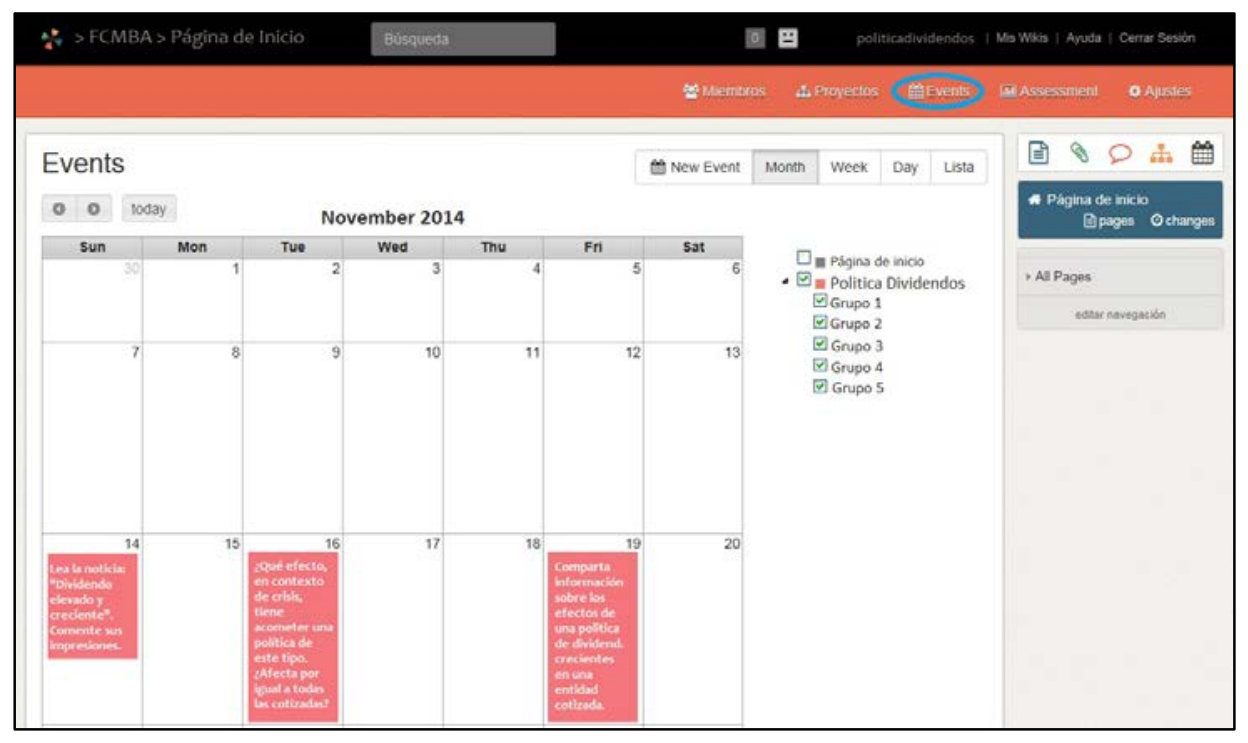

Fig. 8. Crear eventos

Al final del semestre el profesor que ha diseñado la experiencia docente se reúne con sus alumnos para que opinen y hagan una crítica constructiva sobre el desarrollo de la Wiki.

Tal y como manifiestan esta herramienta era ya conocida por la mayoría de los alumnos, siendo el género masculino el que conoce en mayor grado la plataforma.

El alumnado, en todo momento, indica que el proyecto les ha resultado muy interesante y motivador a la hora de aprender los contenidos del área de Finanzas. De forma paralela, el docente efectúa una valoración muy positiva al respecto de la participación de sus alumnos en la experiencia propuesta.

\section{Conclusiones}

Este proyecto tenía como objetivo mejorar el desarrollo de la actividad docente utilizando nuevas metodologías y así poder alcanzar las metas propuestas en la guía académica de una materia vinculada con las Finanzas y dentro del Programa del Máster Business Administration (MBA) en la Facultad de Economía (Universidad de Valencia). Esta experiencia pretender cubrir no solamente las recomendaciones del EEES (Espacio

\section{(c)) EY-NC-ND 2015, Universitat Politècnica de València}


El aprendizaje colaborativo en las Finanzas mediante Wikis. Aplicación práctica a las Políticas de Dividendos.

Europeo de Educación Superior) sino también, a la propia motivación que el docente siente por que sus alumnos aprendan.

Siguiendo en esta línea de trabajo, nos decantamos por los espacios web Wiki como recurso idóneo que responde perfectamente a nuestras necesidades de crear espacios web colaborativos que no requieren de conocimientos informáticos específicos, con un funcionamiento muy intuitivo, fácil y flexible. Esta plataforma permite a sus usuarios interactuar a través de la Wiki y así, compartir todo tipo de conocimientos de carácter gráfico y/o multimedia. Creemos que estos recursos convenientemente utilizados podrían ofrecer un buen instrumento para analizar en qué medida nuestros alumnos están adquiriendo las competencias profesionales necesarias para poder desarrollar su profesión, en un futuro, de manera óptima.

Seleccionamos la plataforma Wikispaces como vía para difundir los contenidos académicos intrínsecamente relacionados con las Finanzas, y en especial, con la Política de Dividendos que las compañías cotizadas llevan a cabo. Apreciamos que esta aplicación práctica ha generado efectos sinérgicos dado que, los alumnos no se han limitado a realizar las actividades propuestas por el docente sino que, adicionalmente añadían al espacio virtual gran variedad de materiales complementarios para ser compartidos por toda la comunidad educativa.

En la última etapa del proyecto, el profesor solicita a sus alumnos que se manifiesten al respecto de la experiencia realizada. Éstos señalan un notable grado de satisfacción con el mismo y sobre todo, la perfecta convivencia entre nuevas tecnologías y la figura del docente como elemento clave para dirigir el proceso de aprendizaje del alumnado.

\section{Referencias}

BARROSO, L. (2009). Creación y Gestión de Wikispace. Centro Multimedia de Información, Sensibilización y Formación para las mujeres.

BEN-ZVI, D. (2007). "Using wiki to promote collaborative learning in statistics education” en Technology Innovations in Statistics Education, 1, 4.

BRENES, E.; GARCÍA, M Y ZARATE, J.P (2012). El aprendizaje colaborativo y el aprendizaje en equipos (Team-Based Learning) en el Desarrollo del Pensamiento Crítico y Creativo.

BRUNS, A. Y HUMPHREYS, S. (2005) "Wikis in teaching and assessment: The M/Cyclopedia project”. International Symposium on Wikis ACM, 25-32.

CHOY, S. Y NG, K. (2007). "Implementing wiki software for supplementing online learning” en Australasian Journal of Educational Techology, 23, 209-226. 
ESTEVE, F. (2009). Bolonia y las TIC: de la docencia 1.0 al aprendizaje 2.0. La cuestión universitaria, 5, 59-68.

FERRO, C.; MARTÍNEZ, A.; Y OTERO, M. (2009). "Ventajas del uso de las TICs en el proceso de enseñanza-aprendizaje desde la óptica de los docentes Universitarios españoles” en Edutec: Revista Electrónica de Tecnología Educativa, 29.

GESER, G. (2007). "Prácticas y recursos de educación abierta: la hoja de ruta OLCOS 2012" en Revista de Universidad y Sociedad del Conocimiento (RUSC), 4, 1.

GUTIERREZ, J.J.; RODRÍGUEZ, M.; BELTRÁN, J.F. Y CONRADI, M. (2012) "Zoowiki: un Proyecto de colaboración Interdepartamental". I jornada de Innovación Educativa. Facultad de Ciencias de la Educación. Universidad de Sevilla.

MUÑOZ, F.; ARVAYO, K.; VILLEGAS, C.; GONZÁLEZ, F. Y SOSA, O. (2014). "El método colaborativo como una alternativa en el trabajo experimental de Química Orgánica” en Educación Química, 25, 4, 464-469.

RODRÍGUEZ, M.J. (2008) “Mi experiencia con las wikis en educación”. II Simposio de Software Libre y Educación.

SÁEZ, J. Y RUIZ, J.M. (2012). "Metodología didáctica y tecnología educativa en el desarrollo de las competencias cognitivas: aplicación en contextos universitarios" en Revista de Currículum y Formación del Profesorado, 16, 3.

SALINAS, J. (1995). Organización escolar y redes: Los nuevos escenarios del aprendizaje. Nuevos canales de comunicación en la enseñanza. Centro de Estudios Ramón Areces, Madrid, 89-117.

SALINAS, J. (1999). "Enseñanza flexible, aprendizaje abierto. Las redes como herramientas para la formación” en Edutec: Revista Electrónica de Tecnología Educativa, 10.

SURIÁ, R. (2010). "Las TIC en las titulaciones universitarias de grado: análisis del conocimiento y uso en el alumnado de la Universidad a Distancia” en Electronic Journal of Reserach in Educational Psychology, 8, 3, 1179-1200.

VILLALVA, M. (2009). Recursos de la Web 2.0 para la Enseñanza de Idiomas. Estudio 2007-2008. 
El aprendizaje colaborativo en las Finanzas mediante Wikis. Aplicación práctica a las Políticas de Dividendos.

ANEXO I: Noticia del periódico Cinco Días en prensa gráfica (13 de octubre de 2014).

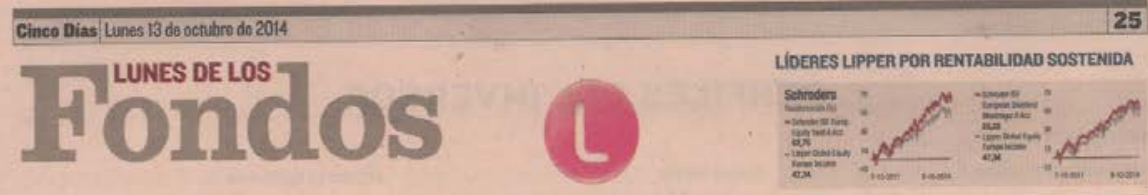

패 gestor de la semana

ALICE GASKELLL Gestora del fondo BGF European Equity Income

\section{Dividendo elevado y creciente}

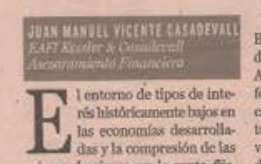
primes de riesgo ent las renta fi

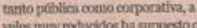
ia expectativa de rementabilidal para It gran mavorta del mervedo de bonos seas modeta en el mejor
lon casos ynoeventid de risogos. Ase

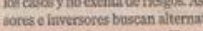
vasy una de ellas, tampoce thre ce niespos, es in de las acciones cose unia

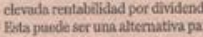
los uhomidors gite buscon

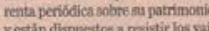

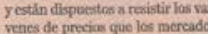
turnitiles Bevia aparjiados.

Esta clase de metivo, la renta we riablecona alto dividendo se tratade una eateguria de imension muty po

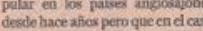
de otros paises europeos $y$ Espati. es todwiá relativamente de menor tamaño. El nemero de fordos en ateguria de Bolks europea oxa at nible para ef mereado espanol ciende a cerra de 40 seggin la hase de catos de Lipper y al fando get cocado por la grotora do cala os que han disfntado de un mej of desempeío en los ultitimos attos in duyendo el presente, cono prost is pitzina

imos con Alice Guskell e

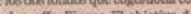
on de acciones eutropets que por milno medio proporcionen an a a de referencia MSCI Europe. demix dicho dividendo ideal nente dobe ser creciente y en par-

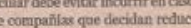

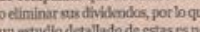
cosirio untes de tomar pasiciono. Este attino aspecto a lis horade an Lare este tipo de fondos as clawe dividentos $n 0$ solo se piende es fientie de ruxlimiento sino que sad it aparrejada de uns cailata de los par

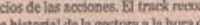
crikente. Adicionalmente, el objir tivo de seloccionar accloues companiss que anmentain avidacenpidanente con un incremeon metio anualizado desde lamza. miento del STh. En los éltimos 12 mesex d dirindendo medio de la cartera ve situib un 45: por encimas del po portionado per lis socioneses en di. dice de réerencix Tambita es i. dos grejen nener mas menor volu.

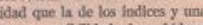
menor sensibilidard a las subididax

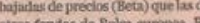

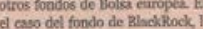

